PROCEEDINGS OF THE

AMERICAN MATHEMATICAL SOCIETY

Volume 127, Number 6, Pages 1597-1603

S 0002-9939(99)05167-9

Article electronically published on February 18, 1999

\title{
AVERAGE ROOT NUMBERS IN FAMILIES OF ELLIPTIC CURVES
}

\author{
OTTAVIO G. RIZZO
}

(Communicated by David E. Rohrlich)

\begin{abstract}
We introduce a height measure on $\mathbf{Q}$ to count rational numbers. Through it, we prove a density result on the average value of the root numbers of families of twists of elliptic curves.
\end{abstract}

Zagier and Kramarz computed in [11] the rank of the curves $x^{3}+y^{3}=m$, with $m$ an integer $<70,000$. These data suggest that the rank is even for exactly half of the twists of $x^{3}+y^{3}=1$. This conjecture has been proved (conditionally on the Birch and Swinnerton-Dyer conjecture) by Mai in [4]. Define, as usual, the root number $W(E)$ of an elliptic curve $E$ as the sign of the functional equation of the $L$ series associated to $E$ (see C.16 of [9]). According to the parity conjecture, $W(E)=(-1)^{\operatorname{rank}(E)}$.

Given a proper definition of average, we can express Mai's result as saying that the average value of the root numbers of the quadratic twists of $x^{3}+y^{3}=1$ is 0 . Let $H(m / n)=\max \{|m|,|n|\}$ be the height of $m / n$, where $m$ and $n$ are relatively prime integers.

Definition. The average value of a function $\psi: \mathbf{Q} \rightarrow \mathbf{R}$ is

$$
\operatorname{Av} \psi(t)=\lim _{T \rightarrow \infty} \frac{\sum_{H(t)<T} \psi(t)}{\sum_{H(t)<T} 1}
$$

where $t$ varies in $\mathbf{Q}$, provided that the limit exists.

Fix throughout this paper an elliptic curve $E: y^{2}=x^{3}+A x+B$ defined over $\mathbf{Q}$, and a polynomial $f(t) \in \mathbf{Q}[t]$. Denote by $E^{f(t)}$ the family of twists $f(t) y^{2}=$ $x^{3}+A x+B$. One would expect Av $W\left(E^{f(t)}\right)$ to be 0 , as in the Zagier-Kramarz case - actually, we have exactly the opposite result:

Theorem 1. Let $E$ be a fixed elliptic curve defined over $\mathbf{Q}$. Given any open subset I of $[-1,1]$, there exists a polynomial $f(t) \in \mathbf{Q}[t]$ of degree at most four, such that Av $W\left(E^{f(t)}\right) \in I$.

To prove this result, we introduce a height measure on $\mathbf{Q}$, and we express the average value of a function as an integral. Using results of Rohrlich [6], we find enough polynomials to realize the conditions of the theorem.

Received by the editors September 15, 1997.

1991 Mathematics Subject Classification. Primary 11G05; Secondary 11D25, 11C08, 28C10.

This research was partially written while the author was supported by a grant of the Istituto Nazionale di Alta Matematica of Rome.

(C)1999 American Mathematical Society 


\section{The Height MEASURE}

Definition. For any subset $U$ of $\mathbf{Q}$, we define its height measure to be the following limit, provided it exists:

$$
\mu(U)=\lim _{t \rightarrow \infty} \frac{\#\{r \in U: H(r) \leq t\}}{\#\{r \in \mathbf{Q}: H(r) \leq t\}} .
$$

If the limit exists, we say that $U$ is $\mu$-measurable.

Remark 2. There exist subsets of $\mathbf{Q}$ which are not $\mu$-measurable.

Recall that a set field is a non-empty family $\Sigma$ of subsets of a set $S$ that contains the empty set, the complement of each element of $\Sigma$, and every finite union of elements of $\Sigma$. Unfortunately, the height measure is not a measure in the classic sense, since it is not $\sigma$-additive: $\mathbf{Q}=\bigcup_{r \in \mathbf{Q}}\{r\}$, but $\mu(\mathbf{Q})=1$, while $\sum_{r} \mu(\{r\})=0$. On the other hand, it is a positive valued additive set function on the set field generated by intervals (see III.1 of [3]).

Proposition 3. Let $U$ and $U^{\prime}$ be $\mu$-measurable subsets of $\mathbf{Q}$. Then the following properties hold:

1. $\mu(\emptyset)=0$;

2. $U \cup U^{\prime}$ is $\mu$-measurable, and $\mu\left(U \cup U^{\prime}\right) \leq \mu(U)+\mu\left(U^{\prime}\right)$, with equality holding if $U$ and $U^{\prime}$ are disjoint;

3. $\mu(\mathbf{Q})=1$.

Proof. Points 1 and 3 follow immediately from the definition of $\mu$, as does point 2 when $U$ and $U^{\prime}$ are disjoint. To prove point 2 in general, one uses the identity $U \cup U^{\prime}=\left(U \backslash U^{\prime}\right) \cup\left(U \cap U^{\prime}\right) \cup\left(U^{\prime} \backslash U\right)$.

Theorem 4. $\mu$ is an additive set function on the set field generated by the intervals in $\mathbf{Q}$. Furthermore, its value is given by

$$
\mu((-\infty, x])= \begin{cases}-\frac{1}{4 x} & \text { if } x \leq-1, \\ \frac{1}{2}+\frac{x}{4} & \text { if }|x| \leq 1, \\ 1-\frac{1}{4 x} & \text { if } x \geq 1 .\end{cases}
$$

Definition. For every integer $t>0$, we define

$$
\begin{gathered}
\Phi(t)=\{r \in \mathbf{Q}: H(r)=t\}, \\
\Phi(t, x)=\{r \in \mathbf{Q}: H(r)=t, r \leq x\} .
\end{gathered}
$$

Lemma 5. If $t>1$, then $\# \Phi(t)=4 \phi(t)$.

Proof. If we write $r=m / n$, with $m$ and $n$ relatively prime integers and with $n>0$, then $\Phi(t)=\left\{m / n:(m, n) \in \mathbf{Z} \times \mathbf{Z}^{>0}, \max \{|m|, n\}=t, \operatorname{gcd}(m, n)=1\right\}$.

If $t>1$, then clearly $\pm t / t \notin \Phi(t)$ and

$$
\begin{aligned}
\Phi(t)=\{ & \pm t / n: n \in \mathbf{Z}, 1 \leq n \leq t, \operatorname{gcd}(n, t)=1\} \\
& \cup\{m / t: m \in \mathbf{Z},-t \leq m \leq t, \operatorname{gcd}(m, t)=1\},
\end{aligned}
$$

whose order is $4 \phi(t)$.

Definition. In analogy to Euler's $\phi$ function, we define for any positive integer $t$ and any positive number $x$, a function

$$
\phi(t, x)=\#\{\text { positive integers } \leq x \text { which are relatively prime to } t\}
$$


and a function

$$
\tilde{\phi}(t, x)=\#\{\text { positive integers }<x \text { which are relatively prime to } t\} .
$$

Proposition 6. Suppose $t>1$. Then

$$
\# \Phi(t, x)= \begin{cases}\phi(t,-t / x) & \text { if } x \leq-1 \\ 2 \phi(t)-\tilde{\phi}(t,-x t) & \text { if }-1 \leq x \leq 0 \\ 2 \phi(t)+\phi(t, x t) & \text { if } 0 \leq x \leq 1 \\ 4 \phi(t)-\tilde{\phi}(t,-t / x) & \text { if } x \geq 1\end{cases}
$$

Proof. Analogously to the proof of Lemma 5, we can rewrite $\Phi(t, x)$ as

$$
\left\{m / n:(m, n) \in \mathbf{Z} \times \mathbf{Z}^{>0}, \max \{|m|, n\}=t, \operatorname{gcd}(m, n)=1, m \leq x n\right\} .
$$

Suppose now that $x \leq-1$; then $m \leq-n$ and $t=H(m / n)=-m$. Thus

$$
\Phi(t, x)=\{-t / n: n \in \mathbf{Z}, \operatorname{gcd}(n, t)=1,1 \leq n \leq-t / x\} .
$$

Hence, $\# \Phi(t, x)=\phi(t,-t / x)$. The other cases are similar.

Proposition 7. For any $x$ and $t>0$ we have that $\left|\phi(t, x)-\frac{x}{t} \phi(t)\right| \leq d(t)$, where $d(n)$ is the number of divisors of $n$. As $T$ increases to infinity,

$$
\sum_{t \leq T} \phi(t, x)=\frac{x}{2 \zeta(2)} T^{2}+O(T \log T)
$$

where the $O$-constant is independent of $x$. The same estimates hold when $\phi$ is replaced by $\tilde{\phi}$.

Proof. By definition, $\phi(t, x)$ is equal to

$$
\phi(t, x)=\sum_{\substack{n \leq x \\ \operatorname{gcd}(n, t)=1}} 1 .
$$

Let $\mu(n)$ be as usual the Möbius function of $n$. We have that, for every positive integer $n$,

$$
\sum_{d \mid n} \mu(d)= \begin{cases}1 & \text { if } n=1 \\ 0 & \text { if } n>1\end{cases}
$$

(See, for example, Theorem 2.1 of [1].) Then

$$
\begin{aligned}
\phi(t, x) & =\sum_{n \leq x} \sum_{d \mid(n, t)} \mu(d)=\sum_{d \mid t} \sum_{\substack{n \leq x \\
n \equiv 0(d)}} \mu(d)=\sum_{d \mid t}\left\lfloor\frac{x}{d}\right\rfloor \mu(d) \\
& =\sum_{d \mid t} \frac{\mu(d)}{d} x-\sum_{d \mid t} \mu(d)\left(\frac{x}{d}-\left\lfloor\frac{x}{d}\right\rfloor\right) .
\end{aligned}
$$

It is well known (see Theorem 2.3 of [1]) that $\sum_{d \mid t} \mu(d) / d=\phi(t) / t$. Therefore, since

$$
\left|\mu(d)\left(\frac{x}{d}-\left\lfloor\frac{x}{d}\right\rfloor\right)\right| \leq 1,
$$

we have that

$$
\left|\phi(t, x)-\frac{\phi(t)}{t} x\right| \leq d(t)
$$


Analogously, we have that

$$
\tilde{\phi}(t, x)=\sum_{d \mid t} \sum_{\substack{n<x \\ n \equiv 0(d)}} \mu(d)=\sum_{d \mid t} \frac{\mu(d)}{d} x+\sum_{d \mid t} \mu(d)\left(\left\lceil\frac{x}{d}\right\rceil-\frac{x}{d}-1\right) .
$$

Once again we get

$$
\left|\mu(d)\left(\left\lceil\frac{x}{d}\right\rceil-\frac{x}{d}-1\right)\right| \leq 1
$$

thus

$$
\left|\tilde{\phi}(t, x)-\frac{\phi(t)}{t} x\right| \leq d(t) .
$$

The second part follows at once from the following formulae, as $T$ tends to infinity (see Theorems $3.3,11.7$, and 3.7 of [1]):

$$
\begin{gathered}
\sum_{n \leq T} d(n)=T \log T+O(T), \\
\sum_{n \leq T} \phi(n)=\frac{1}{2 \zeta(2)} T^{2}+O(T \log T) .
\end{gathered}
$$

Proof of Theorem 4. Suppose that eq. (1) holds: it follows immediately that intervals are $\mu$-measurable. By Proposition 3, all finite combinations of intervals are $\mu$-measurable. Thus, $\mu$ is additive on the set field generated by intervals.

We are left to prove eq. (1): we have that

$$
\lim _{T \rightarrow \infty} \frac{\#\{r \in \mathbf{Q}: H(r) \leq T, r \leq x\}}{\#\{r \in \mathbf{Q}: H(r) \leq T\}}=\lim _{T \rightarrow \infty} \frac{\sum_{t=1}^{T} \# \Phi(t, x)}{\sum_{t=1}^{T} \# \Phi(t)} .
$$

Suppose that $x \leq 1$. Then, by Lemma 5 and Proposition 6 ,

$$
\lim _{T \rightarrow \infty} \frac{\sum_{t=1}^{T} \# \Phi(t, x)}{\sum_{t=1}^{T} \# \Phi(t)}=\lim _{T \rightarrow \infty} \frac{\sum_{t=1}^{T} \phi(t,-t / x)}{\sum_{t=1}^{T} 4 \phi(t)} .
$$

By Proposition 7 and eq. (2), this is

$$
=\lim _{T \rightarrow \infty} \frac{\sum_{t=1}^{T}\left(-\phi(t) \frac{1}{x}+O(d(t))\right)}{\sum_{t=1}^{T} 4 \phi(t)}=-\frac{1}{4 x}+O\left(\frac{T \log T}{\sum_{t=1}^{T} \phi(t)}\right) .
$$

By eq. (3), the error term is actually $O(\log T / T)$, so that the limit converges and $\mu(x)=-1 / 4 x$. The remaining cases are proved in a similar way.

Definition. The height measure $\mu$ of $\mathbf{R}$ is the measure induced on the Borel sets of $\mathbf{R}$ by the function $\mu(x)$.

Remark 8. Since $\mu(x)$ is a bounded monotone increasing continuous function, differentiable at every $x \neq \pm 1$, by standard measure theory $\mu$ is well defined (see, for example, Chapter 7 of [8], in particular Exercise 13), and it is absolutely continuous with respect to the standard Lebesgue measure.

Proposition 9. Let $f$ be a step function over $\mathbf{Q}$, i.e., $f=\sum_{i=0}^{n} a_{i} \chi_{i}$, where $a_{i} \in \mathbf{Q}$ and $\chi_{i}$ is the characteristic function of an interval. Then $\operatorname{Av} f(t)=\int_{\mathbf{R}} f(t) \mathrm{d} \mu(t)$. 
Proof. If $\chi$ is the characteristic function of some interval $I$, then it is clear that

$$
\operatorname{Av} \chi(t)=\lim _{T \rightarrow \infty} \frac{\#\{r \in \mathbf{Q}: H(r) \leq T, r \in I\}}{\#\{r \in \mathbf{Q}: H(r) \leq T\}}=\mu(I)=\int_{\mathbf{R}} \chi(t) \mathrm{d} \mu(t) .
$$

By linearity, we get the same result for $f$.

\section{Root NUMBers}

Recall that the root number of an elliptic curve has an intrinsic definition (see $[2],[10]$ and especially [7]) independent of any conjectures, as a product of local factors.

Definition. Given an elliptic curve $E$ and a polynomial $f(t)$, we say that $f(t)$ is a Rohrlich polynomial for $E$ if, for every $t$ such that $f(t) \neq 0, W\left(E^{f(t)}\right)=\epsilon \operatorname{sgn} f(t)$, where $\epsilon=1$ is independent of $t$.

Proposition 10. Let $m, n$ be even integers with $0 \leq m \leq n$. If $E$ does not satisfy the technical condition (§) of [6], we further suppose that $n$ is divisible by 4. Then there exists an irreducible polynomial $f(t) \in \mathbf{Q}[t]$, Rohrlich for $E$, of degree $n$, and exactly $m$ real zeros, all of them simple.

Proof. See Proposition 8 of [6].

Notation. Given a real function $f(t)$, write $s_{f}(t)$ for the function $\operatorname{sgn} f(t)$.

Proposition 11. Let $f$ be a Rohrlich polynomial for $E$ of even degree $n$. For any rational number $r \neq 0$, define $f_{r}(t)=r^{n} f(t / r)$. Then $f_{r}(t)$ is a Rohrlich polynomial for $E$.

Proof. Since $n$ is even, $f_{r}(t) \equiv f(r / t) \bmod \mathbf{Q}^{\star 2}$. Hence,

$$
W\left(E^{f_{r}(t)}\right)=W\left(E^{f(t / r)}\right)=\operatorname{sgn} f(t / r)=\operatorname{sgn} f_{r}(t / r) .
$$

Lemma 12. Suppose $f(t)$ is a Rohrlich polynomial for $E$. Then, for every $r \in \mathbf{Q}$, $g(t)=f(t-r)$ is Rohrlich.

Proof. Obvious.

Proposition 13. Let $f$ be a polynomial with real coefficients, even degree, and an even number of real roots. Let $\epsilon=\lim _{|t| \rightarrow \infty} s_{f}(t)$. Define a map

$$
\begin{aligned}
\lambda: \mathbf{R} & \longrightarrow \mathbf{R} \\
r & \longmapsto \int_{\mathbf{R}} s_{f_{r}}(t) \mathrm{d} \mu(t) .
\end{aligned}
$$

Assume that $\epsilon f(0)<0$. Then $\lambda(\mathbf{Q})$ is dense in $[-1,1]$.

Proof. The idea is to prove that:

1. $\lim _{r \rightarrow \infty} \lambda(r)=\epsilon$.

2. $\lim _{r \rightarrow 0} \lambda(r)=s_{f}(0)$.

3. $\lambda$ is a continuous function from $\mathbf{R}$ to $\mathbf{R}$. 
Thus, if the two limits are +1 and -1 , then $\lambda(r)$ spans $[-1,1]$ as $r$ runs from 0 to $\infty$. Restricting $r$ to $\mathbf{Q}$ leaves the image of $\lambda$ dense.

Let $x_{1}, \ldots, x_{m}$ be the real roots of $f$, and let $x_{0}=-\infty, x_{m+1}=+\infty$. By assumption $f(0) \neq 0$, say $x_{i_{0}}<0<x_{i_{0}+1}$. Let $\chi_{i}$ be the characteristic function of $\left(x_{i}, x_{i+1}\right)$. Since we assumed that $f$ has even degree and an even number of real roots, we can decompose $s_{f}(t)=\sum_{i=0}^{m}(-1)^{i} \epsilon \chi_{i}(t)$. Since $s_{f_{r}}(t)=s_{f}(t / r)$,

$$
\lim _{r \rightarrow \infty} \int_{\mathbf{R}} s_{f_{r}}(t) \mathrm{d} \mu(t)=\epsilon \sum_{i=0}^{m}(-1)^{i} \lim _{r \rightarrow \infty} \int_{\mathbf{R}} \chi_{i}(t / r) \mathrm{d} \mu(t),
$$

where we can exchange the limit with the sum, since the latter is finite. By Theorem 4 ,

$$
\lim _{r \rightarrow \infty} \int_{\mathbf{R}} \chi_{0}(t / r) \mathrm{d} \mu(t)=\lim _{r \rightarrow \infty} \mu\left(x_{0} / r\right)-\mu(-\infty)=\mu(0)-\mu(-\infty)=\frac{1}{2} .
$$

Analogously,

$$
\lim _{r \rightarrow \infty} \int_{\mathbf{R}} \chi_{m}(t / r) \mathrm{d} \mu(t)=\frac{1}{2} .
$$

On the other hand, for any $i=1, \ldots, m-1$,

$$
\lim _{r \rightarrow \infty} \int_{\mathbf{R}} \chi_{i}(t / r) \mathrm{d} \mu(t)=\lim _{r \rightarrow \infty} \mu\left(x_{i+1} / r\right)-\mu\left(x_{i} / r\right)=0 .
$$

Putting (4), (5) and (6) together, we have proved that

$$
\lim _{r \rightarrow \infty} \int_{\mathbf{R}} s_{f_{r}}(t) \mathrm{d} \mu(t)=\epsilon
$$

Consider now the same problem as $r$ decreases to 0 . If $i \neq i_{0}$, then $\chi_{i}(t / r) \rightarrow 0$; on the other hand, $\chi_{i_{0}}(t / r) \rightarrow 1$. Hence,

$$
\lim _{r \rightarrow 0} \int_{\mathbf{R}} \chi_{i}(t / r) \mathrm{d} \mu(t)= \begin{cases}1 & \text { if } i=i_{0}, \\ 0 & \text { otherwise. }\end{cases}
$$

Therefore,

$$
\lim _{r \rightarrow 0} \int_{\mathbf{R}} s_{f_{r}}(t) \mathrm{d} \mu(t)=s_{f}(0)
$$

We are left to prove the continuity of $\lambda$. Since $s_{f}$ is a finite linear combination of characteristic functions of intervals, it is enough to prove that the map

$$
r \longrightarrow \int_{\mathbf{R}} \chi_{(a, b)}(t / r) \mathrm{d} \mu(t)=\int_{a r}^{b r} \mathrm{~d} \mu(t)=\mu(b r)-\mu(a r)
$$

is continuous for any $-\infty \leq a \leq b \leq \infty$, where $\chi_{(a, b)}$ is the characteristic function of $(a, b)$. Since $\mu$ is bounded and $\mu(t)$ is continuous by Theorem 4 , we are done.

Proof of Theorem 1. By Proposition 10, we can choose a polynomial $f$ of degree two or four, with exactly two real roots $x_{1}<x_{2}$, both simple. By Lemma 12, we can suppose that $x_{1}<0<x_{2}$. By Propositions 9 and 11,

$$
\text { Av } W\left(E^{f_{r}(t)}\right)=\int_{\mathbf{R}} s_{f_{r}}(t) \mathrm{d} \mu(t) .
$$

But $f$ satisfies the conditions of Proposition 13, and this proves the statement of the theorem. 


\section{EXAMPLE}

Let $E$ be the modular curve $X_{0}(11): y^{2}+y=x^{3}-x^{2}-10 x-20$, and let $f(t)=$ $11-t^{2}$. It is shown in 4.2 .1 of [5], using the machinery of [6], that $W\left(E^{f(t)}\right)=$ $-\operatorname{sgn} f(t)$.

Proposition 14. If $E$ and $f$ are as above, we have that:

1. For any $r \in \mathbf{Q}, r>0$,

$$
\operatorname{Av}_{t} W\left(E^{f_{r}(t)}\right)= \begin{cases}1 /(r \sqrt{11})-1 & \text { if } r \sqrt{11}>1, \\ 1-r \sqrt{11} & \text { if } r \sqrt{11}<1 .\end{cases}
$$

2. The set $\left\{\operatorname{Av}_{t} W\left(E^{f_{r}(t)}\right): r \in \mathbf{Q}, r>0\right\}$ is dense in $[-1,1]$.

Proof. As in eq. (7), we have that

$$
\operatorname{Av}_{t} W\left(E^{f_{r}(t)}\right)=\int_{\mathbf{R}}-\operatorname{sgn}\left(11 r^{2}-t^{2}\right) \mathrm{d} \mu(t),
$$

since the roots of $11 r^{2}-t^{2}$ are $t=\sqrt{11} r$, this is

$$
=1+2 \mu(-r \sqrt{11})-2 \mu(r \sqrt{11}) .
$$

Item 1 now follows from Theorem 4. Item 2 follows either from 1 or from Theorem 1.

\section{REFERENCES}

[1] Tom M. Apostol, Introduction to analytic number theory, Undergraduate Texts in Mathematics, Springer-Verlag, New York, 1976. MR 55:7892

[2] Pierre Deligne, Les constantes des quations fonctionnelles des fonctions L, Modular functions of one variable, II, Lecture Notes in Math. 349, Springer-Verlag, Berlin, 1973, pp. 501-597. MR 58:22020

[3] Nelson Dunford and Jacob T. Schwarz, Linear operators, part I, Wiley, New York, 1988. MR 90g:47001a

[4] Liem Mai, The average analytic rank of a family of elliptic curves, J. Number Theory 45 (1993), 45-60. MR 95d:11080

[5] Ottavio G. Rizzo, On the variations of root numbers in families of elliptic curves, Ph.D. thesis, Brown University, Providence, RI, 1997.

[6] David E. Rohrlich, Variation of the root number in families of elliptic curves, Compos. Math. 87 (1993), no. 2, 119-151. MR 94d:11045

[7] _ Elliptic curves and the Weil-Deligne group, Elliptic Curves and Related Topics (Hershy Kisilevsky and M. Ram Murty, eds.), CRM Proceedings \& Lecture Notes, vol. 4, Centre de Recherches Mathématiques, Amer. Math. Soc., 1994, pp. 125-157. MR 95a:11054

[8] Walter Rudin, Real and complex analysis, second ed., McGraw-Hill, 1974. MR 49:8783

[9] Joseph H. Silverman, Advanced topics in the arithmetic of elliptic curves, GTM 151, SpringerVerlag, New York, 1994. MR 96b:11074

[10] John Tate, Number theoretic background, Automorphic Forms, Representations and LFunctions, part 2, Proc. Sympos. Pure Math., XXXIII, Amer. Math. Soc., Providence, R.I., 1977, pp. 3-26. MR 80m:12009

[11] Don Zagier and Gerhard Kramarz, Numerical investigations related to the L-series of certain elliptic curves, J. Indian Math. Soc. 52 (1987), 51-69. MR 90d:11072

Department of Mathematics, Brown University, Box 1917, Providence, Rhode Island 02912

Current address: Department of Mathematics and Statistics, Queen's University, Kingston, Ontario, Canada K7L 3N6

E-mail address: otto@math.brown.edu 